\title{
Reconocimiento: una característica de las prácticas pedagógicas en relatos de maestros de escuelas normales superiores en contextos de conflicto interno en Colombia*
}

\author{
Milton Molano Camargo a \\ Felipe Andrés Aliaga Sáez ${ }^{b}$
}

\section{Resumen}

Este artículo tiene como objetivo dar a conocer los significados de las prácticas pedagógicas narradas por maestros de escuelas normales superiores situados en contextos educativos impactados por el conflicto armado interno colombiano. La metodología empleada fue de tipo cualitativo con un enfoque hermenéutico desde la narrativa. El grupo de estudio fueron 17 profesores de siete regiones distintas en Colombia afectadas por el conflicto interno armado colombiano. Las prácticas pedagógicas como lugar de reconocimiento, en la perspectiva ricoeuriana, es una dimensión central que se describe en este trabajo. Se trata de la categoría más relevante que emerge en la mayoría de los relatos de los profesores y las profesoras y que está ligada a la de capacidad y configuración de la identidad. El profesor se forma a sí mismo a través de la práctica, que le permite declararse capaz de hacer, de lograr, de transformar, de liderar, y sentirse depositario de la misión de educar.

Palabras clave: Reconocimiento. Práctica Pedagógica. Investigación Narrativa. Pedagogía Narrativa.

\footnotetext{
* Este artículo forma parte de la tesis doctoral denominada Narrativas de las prácticas pedagógicas en contextos impactados por el conflicto interno colombiano. Relatos de maestros de escuelas normales superiores, en el programa doctoral Educación y Sociedad de la Universidad de La Salle en Bogotá, Colombia (MOLANO, 2019).

a Universidad de La Salle, Bogotá, Colombia.

b Universidad de La Salle, Bogotá, Colombia.
} 


\section{Introducción}

El abordaje de las prácticas pedagógicas se ha convertido en un eje recurrente en la reflexión pedagógica contemporánea. Gracias al aporte de las ciencias sociales y la filosofía, la mirada centrada en un hacer instrumental, cuantificable y meramente empírico (VENÄLÄINEN, 2009) se ha enriquecido para dar paso a comprensiones más amplias, complejas, que permiten abordarla en sus contextos, en los discursos y en las reflexiones críticas que muestran su potencial para abordarla como un núcleo para pensar lo pedagógico, el maestro, la escuela, y la formación. Esto la hace una categoría de gran importancia para desarrollar ejercicios investigativos.

Y es en medio de este movimiento por la práctica (GAJARDO-ASBUN, 2019; VANDEYAR, 2020), y además por sus implicaciones en el aprendizaje de los estudiantes, en su desarrollo y en la calidad de vida de las comunidades, sobre todo de las asediadas por el conflicto armado colombiano, que interesa ahondar en su comprensión. Igualmente, hay un fértil valor en asumir la categoría de práctica como campo de conocimiento pedagógico, por cuanto permite construir desde las tensiones epistémicas y metodológicas entre lo individual, lo colectivo y lo social; la reproducción del sistema y las posibilidades de transformarlo; lo cotidiano y el horizonte de vida; el sentido y la inteligibilidad; lo que acontece y el acontecimiento (ZULUAGA GARCÉS, 2005); el discurso que constituye y el discurso que se construye. Sin embargo, esta categoría tan amplia en su desarrollo necesita ser acotada para un abordaje más profundo y centrado, razón por la cual el estudio de las prácticas pedagógicas se hizo desde sus comprensiones como acción (KEMMIS, 2007; RESTREPO, 2006), discurso (ZULUAGA GARCÉS, 2005) y relato (ROSIEK; ATKINSON, 2007), esta última como una manera alternativa de comprenderla en cuanto busca un diálogo entre las dos primeras.

Por estas razones, es importante explorar otras posibilidades de comprensión de la práctica pedagógica que puedan identificar lo mejor de los aportes teóricos hasta ahora desarrollados, en una síntesis que permita investigar las prácticas pedagógicas de los maestros en situaciones de violencia y destrucción como las que se viven en Colombia (ZULUAGA NIETO, 2012). De esta manera, se responde al siguiente interrogante: ¿qué significados en torno a las prácticas pedagógicas emergen de las narraciones de los maestros de escuelas normales superiores situados en contextos educativos impactados por el conflicto armado interno colombiano?

En consecuencia, se parte de relatos de maestros de escuelas normales superiores que están en contextos afectados por el conflicto interno colombiano para plantear 
formas de compresión de las prácticas pedagógicas en las que es posible encontrar sujeto, intencionalidad y un modo propio de construcción, a manera de una gramática interpretativa.

El artículo se estructura en tres partes: la primera plantea algunas reflexiones que fundamentan el estudio; la segunda explica la metodología; y la tercera propone los resultados en torno a la categoría de reconocimiento como constructo emergente, presente en las narraciones de todos los maestros.

\section{Referentes conceptuales del estudio}

La investigación narrativa ha adquirido cada vez mayor relevancia en los últimos años. En el campo anglosajón, ha sido destacado el trabajo de Jean Clandinin y Michel Conelly y su grupo de la Universidad de Alberta y la Universidad de Toronto en Canadá, quienes han asumido un claro liderazgo en la circulación de saberes sobre investigación narrativa, por ejemplo, a través del Handbook of Narrative Inquiry. Clandinin y Rosiek (2007) que plantea tres lugares comunes de la investigación narrativa. El primero la temporalidad, "In narrative inquiry it is important to always try to understand people, places, and events as in process, as always in transition" (CLANDININ; ROSIEK, 2007, p. 24). El segundo, la socialización, es decir, las historias de los sujetos están en contextos y se construyen en interacciones personales. El tercero, el lugar, es decir "the specific concrete, physical and topological boundaries of place or sequence of places where the inquiry and events take place" (CLANDININ; ROSIEK, 2007, p. 25).

En este marco, se destacan los trabajos sobre la identidad del profesor, que en tiempos de transformaciones tan rápidas y radicales como las que vivimos actualmente han adquirido importancia. Un reciente estado del arte sobre identidad docente (GAJARDO-ASBUN, 2019) muestra que un $75 \%$ de las investigaciones en esta área se hace en marcos metodológicos de corte narrativo. Uno de los aspectos que se repiten en estos ejercicios de investigación es la necesidad de problematizar y abordar la identidad para lo cual se hacen exhaustivos recorridos teóricos, por ejemplo, en la definición del concepto, los lugares del yo, y cuestiones ligadas a los campos de: agencia, emoción, narrativa y discurso, papel de la reflexión, e influencia de los factores contextuales y de las experiencias previas a la formación inicial (BEAUCHAMPA; THOMAS, 2009; MORTOLA, 2006). Caben aquí también las investigaciones sobre los ciclos de vida profesional de los profesores que intentan descubrir regularidades y etapas reiterativas en las vidas de los docentes (BOLÍVAR, 1997). 
En el mundo anglosajón, aparecen los temas interculturales y los relatos de identidad en contextos distintos al propio, o en situaciones de conflicto histórico (FERGUSON, 2011; TULVISTE, 2011). Además, el uso de la narrativa como herramienta en la comprensión de la identidad como orientación moral, desde los estudios del filósofo canadiense Charles Taylor y aplicados a los juicios que los profesores hacen sobre la "bondad" de los estudiantes (HOWARD, 2008).

El camino narrativo muestra cómo el conocimiento se forma en las situaciones experimentadas por el profesor, que se construye y reconstruye en la medida en que el docente vive sus historias, las relata y las revive a través de la reflexión suscitada en el relato, de tal forma que hay una especie de discurso narrativo (OLSHTAIN; KUPFERBERG, 1998). Como discurso es analizado también desde su lugar de enunciación y su relación con el poder a través de las redes sociales en el que se moviliza (NESPOR; BARYLSKE, 1991).

En relación con la práctica pedagógica, una vía de diálogo para acercarse a desarrollar una categoría de práctica pedagógica desde una perspectiva situada en la experiencia, en el acontecimiento, es la que sugiere el concepto de praxis como configuración narrativa de la acción, planteado por el filósofo francés Paul Ricoeur, y que podría entenderse como un diálogo fecundo entre el discurso y la acción, es decir, como un camino de acercamiento entre lo deconstructivo y lo constructivo. Con respecto al sujeto, es importante decir que la práctica pedagógica contemplaría un sujeto con rostro e historia y que construye una identidad personal que está marcada por la temporalidad como ya se ha mostrado a lo largo del texto. Así, los referentes clave son las capacidades y el reconocimiento. Ricoeur establece una valiosa tipología de las capacidades básicas "en la unión de lo innato y de lo adquirido" pues "la persona es su historia" (2004, p. 1). De tal forma que las prácticas pedagógicas necesitan ser comprendidas en el relato del maestro, en la configuración narrativa de la experiencia.

El esquema de tipología de estas capacidades es: la capacidad de decir, de actuar y contar, a las que se agregan la imputabilidad y la promesa. "El acento principal se desplaza de un polo a primera vista moralmente neutro, a un polo explícitamente moral donde el sujeto capaz se prueba como sujeto responsable" (RICOEUR, 2004, p. 1).

Si se mira para el maestro, poder decir es producir un discurso coherente que se dice a alguien con sentido conforme a reglas comunes, que tendría que ver con los campos disciplinares y pedagógicos del oficio. Poder actuar, es en medio de lo contingente, de lo imprevisto, tener la capacidad de producir acontecimientos en 
la sociedad, es decir, intervenir, generar proyectos educativos que tienen impactos sobre las comunidades. Poder narrar la experiencia es una capacidad con lugar prominente, pues los acontecimientos solo se vuelven legibles e inteligibles cuando se cuentan en una historia, cuando se configura narrativamente la vida. La imputabilidad y la promesa son capacidades claramente morales, que vuelven al agente responsable, capaz de atribuirse una parte de las consecuencias de la acción. "Sobre esta base la promesa es posible; el sujeto se compromete con su palabra y dice que hará mañana lo que dice hoy: la promesa limita lo imprevisible del futuro, a riesgo de traición" (RICOEUR, 2004, p. 2).

Aunque estas capacidades no implican reconocimiento por parte de otros, pues la certeza de hacer algo es íntima, cada una de ellas requiere un interlocutor, que en el mundo educativo haría referencia a los demás actores del proceso. Es decir, un sujeto capaz de responder, de iniciar un diálogo con otros maestros, los estudiantes, los padres de familia, las comunidades locales. El poder actuar implica que la acción es con otros, la historia de vida (el relato) se compone de una multitud de otras historias que se entrecruzan. La imputabilidad vuelve al sujeto responsable ante los demás y, por último, la promesa requiere de un testigo que la recibe y la registra. "Sin embargo, lo que les falta a estas implicaciones de los demás en la certeza privada de poder hacer, es la reciprocidad, la mutualidad que son las únicas que permiten hablar de reconocimiento en el mejor sentido" (RICOEUR, 2004, p. 2); así, la práctica pedagógica adquiere un carácter claramente ético y político.

Ricoeur entiende que el reconocimiento se ha dado en una lucha, en una especie de combate por la búsqueda de un lugar, aunque cuestiona esa dinámica y se pregunta "si no existe también, desde el origen, una especie de benevolencia vinculada con la similitud de hombre a hombre en la gran familia humana" (RICOEUR, 2004, p. 4). Las búsquedas de los seres humanos entrañan una petición de reconocimiento que es insaciable y fuente de conflicto. Pero el reconocimiento de manera pacífica y solidaria también es un hecho que se puede experimentar.

\section{Metodología}

El interés de este trabajo se centró en la interpretación de la práctica pedagógica en narrativas de maestros que trabajan en escuelas normales superiores, instituciones que forman maestros para la Educación Básica, ubicadas en zonas de conflicto interno colombiano. Partió de comprender que la configuración narrativa ofrece un puente entre el discurso y la acción y permite abordar la tensión entre el horizonte y el ejercicio de la cotidianidad, además de establecer un diálogo entre el sujeto individual, los otros y el contexto. 
Por tal razón, en este estudio se adoptó el enfoque cualitativo en su dimensión hermenéutica que busca interpretar, comprender y dar significado al mundo de la vida y a la acción humana. Desde esta perspectiva, el lenguaje, configura y estructura la experiencia vivida, convirtiéndose en el medio fundamental para la compresión de la realidad. De ahí que, la narrativa (ROSIEK; ATKINSON, 2007) se ve como una manera de reconocer lo subjetivo; porque es a partir del lenguaje que el sujeto re-construye la realidad social.

En ese sentido, este trabajo utilizó una perspectiva narrativa en su método (QUINTERO, 2018), es decir, en la configuración de un propósito, unos fundamentos conceptuales y epistémicos y unas maneras de proceder, y en cuanto a los dispositivos fundamentales de recolección de la información que son los relatos de los maestros, así como en unas decisiones respecto al análisis, la presentación de los resultados y por supuesto las conclusiones.

Para efectos del presente estudio, el uso de la investigación narrativa buscó hacer visibles aspectos de las subjetividades en las biografías de profesores y profesoras que trabajan en escuelas normales ubicadas en zonas afectadas por el conflicto interno colombiano, que permiten comprender aspectos de las prácticas pedagógicas que no pueden ser vistas en su profundidad desde otras perspectivas de investigación educativa. Se trata de asumir el relato en la importancia que tiene como fenómeno que constituye a la persona en una dinámica identidad narrativa (RICOEUR, 1996).

En la continuidad, las experiencias se van concatenando y se comunican unas con otras, hay un curso de acción que permite a quien construye la historia ver un continuum de la vida que tiene un horizonte que se tensiona y resignifica con base en lo que acontece. "En cualquier lugar, un punto de vista sobre sí mismo, desde este continuo, ya sea imaginado en el presente, en el pasado o en el futuro, tiene como base una experiencia en el pasado y se orienta hacia una experiencia futura" (MOLANO; BAQUERO, 2009, p. 125).

El grupo de estudio estuvo integrado por dieciséis (17) maestros y maestras de ocho (8) Escuelas Normales Superiores (ENS) situadas en zonas donde la situación de vulnerabilidad para el posconflicto es extrema o alta (VALENCIA; AVIA, 2016), cruzadas con siete nodos regionales según la organización geográfica de estas instituciones a lo largo y ancho de Colombia. Las ENS están organizadas bajo la Asociación Nacional de Escuelas Normales Superiores (Asonen) que se estructura en siete nodos regionales que las agrupan. El acceso a este grupo se logró por medio de los criterios de diversidad territorial, género y años de experiencia docente. 
En cuanto al desarrollo de la investigación se procedió por fases como sigue:

- Producción de relatos de la práctica pedagógica de profesores de escuelas normales superiores que trabajan en sectores impactados por el conflicto interno: Esta producción se realizó a través del instrumento denominado por Atkinson (2007) entrevista sobre la propia vida, entre julio de 2017 y enero de 2018. Son una forma subjetiva e ideográfica de expresar cada momento de la vida de una persona como un todo y de transmitir el significado de cada uno de ellos.

- Análisis de los relatos: se adaptó la estrategia de procesamiento de la información propuesta por Quintero (2018), la cual está conformada por 4 momentos:

Momento 1: registro de transcripción y codificación que buscó identificar un acontecimiento que tiene trama narrativa, es decir quin narra hace algo a lo cual le otorga valor en una red de significaciones que se relacionan con un contenido, un lugar, un tiempo y un modo de la acción.

Momento 2: nivel textual. Pre-concepción de la trama narrativa. Se procedió a agrupar los fragmentos por cada uno de los códigos emergentes, de tal manera que fue posible identificar recurrencias que permitieron identificar tópicos narrativos respecto a los objetivos.

Momento3: nivel Contextual de la trama narrativa; se trató del ejercicio descriptivo que evidencia la interpretación de los nodos narrativos identificados.

Momento 4: nivel metatextual Reconfiguración de la trama narrativa, es decir, la construcción de un nuevo relato integrador que asume la polifonía de las voces y que traza una gramática de las prácticas pedagógicas. y que hace una propuesta metodológica desde la perspectiva ricoeuriana (2000) de la triple mímesis. Los análisis se realizaron con el soporte del software cualitativo MAXQDA.

\section{Resultados}

Las prácticas como lugar del reconocimiento son una de las categorías emergentes más importantes que resultan de la investigación y que aparece en todos los objetivos. En este caso, tiene que ver con la declaración del sujeto capaz en su ser, su hacer y saber, alguien que produce transformación, que puede tocar el corazón de los estudiantes, de hacer del mundo un lugar distinto, de asumir un 
liderazgo y tener una voz que mueve a otros. Y en esto el estudiante, el egresado, el padre de familia opera como el testigo que da fe, que constituye al profesor como depositario de una misión. Esto se evidencia desde tres aspectos:

\subsection{El maestro como sujeto capaz}

Los relatos de los profesores y las profesoras evidencian conciencia de sus capacidades, de forma que hay un descubrirse a sí mismos en la práctica; esto se asocia a las dimensiones del ser, el saber y el hacer, y del liderazgo como una capacidad central e integradora.

Uno de los rasgos más recurrentes en los relatos es el que tiene que ver con el ser del maestro, con sus valores o cualidades personales; a este respecto está lo que podría denominarse un sentido humano, inspirado algunas veces por motivos morales o religiosos que los hace aptos por su capacidad de escucha, por su sensibilidad, por ser capaz de "ponerse en los zapatos de otro", por ser ejemplo de comportamiento para trabajar en la parte vocacional de los jóvenes que se forman para ser maestros, "Yo me siento guardiana de la palabra, me eligieron por eso, porque dijeron sí, tú tienes esa forma de llegar, de comunicarte. Es una fortaleza que tengo" (Mujer, Uribia-Guajira, entrevista 3). Otro aspecto es el de una actitud de permanente aprendizaje, una apertura a estar en formación constante "el que está leyendo, se está actualizando, siempre soy ese, y vengo y oriento eso en mis estudiantes" (mujer, Icononzo-Tolima, entrevista 15).

Hay también cierto sentimiento de búsqueda de perfección en el hacer, así haya que hacer sacrificios personales, "soy una persona que vive intensamente porque no soy educadora en la escuela, soy una educadora, mujer, madre, esposa, que se piensa la vida desde esos lugares, y eso obviamente me trae muchísimas felicidades, para mí la Educación es un motor" (mujer, Riosucio-Caldas, entrevista 13). Por último, hay también reconocimiento respecto a los valores del territorio y la etnia: "Eso somos los indígenas, buscamos siempre, la valoración del territorio, la valoración del espacio, yo estaba muy bien por allá, pero siempre era como ¿y mi comunidad qué?, ¿mi espacio qué?” (hombre, Riosucio-Caldas, entrevista 14).

Respecto al saber sobre el hacer, el conocimiento es un aspecto diferenciador que le permite a los profesores y profesoras lograr cosas importantes, como los nombramientos en escuelas públicas y como el acceso y el éxito educativo "Siempre estuve entre los primeros lugares en los semestres. La perspectiva mía como estudiante era distinta" (hombre, Uribia-Guajira, entrevista 4). También alcanzar reconocimiento profesional al ganar proyectos y programas impulsados 
desde distintas organizaciones: "Un proyecto que fue significativo, lo presenté al "Premio Compartir" y allá fue reconocido, estuve entre los nominados a maestro ilustre" (hombre, San Juan Nepomuceno-Bolívar, entrevista 12).

Frente al hacer, las habilidades son reconocidas como innatas, adquiridas en el ambiente familiar y son vistas como herramientas para la práctica "debido a unas habilidades que yo tengo, se me es fácil la artística, algo como de mi familia. Entonces, me pusieron a dar artística en todos los grados" (mujer, Istmina-Chocó, entrevista 1).

Del mismo modo, están las habilidades dadas por el gusto personal y como una mediación para vincular otras dimensiones del oficio, "Siempre he sido deportista. Entonces, me gustaba mucho la parte de la lúdica, y a través de la lúdica fui insertando los otros conocimientos" (hombre, Icononzo-Tolima, entrevista 15).

Frente a las comunidades, el liderazgo es más evidente en el rol que juega el profesor de la escuela rural, que tiende a asumir distintas responsabilidades y se le confiere una voz de autoridad del saber: "El profesor es el personaje de la vereda, al que tienen que consultar, el que convoca a la junta de padres de familia, la junta de acción comunal. En la zona rural la vida gira en torno al campo y la escuela. En todos los eventos tiene que estar el profe" (hombre, San Juan Nepomuceno-Bolívar, entrevista 12).

Respecto a los compañeros docentes, el liderazgo es posicionamiento como resultado del trabajo del profesor hacia dentro y hacia fuera de la institución. Viene en forma de responsabilidades y de posibilidad de influencia a través de una voz que es escuchada, y aceptada, "Trabajo el programa radial de los maestros. Se me han acercado varios profes, varias madres de familia, los muchachos del nocturno me dicen: usted excelente en esa emisora, nosotros lo escuchamos, me saludan y todo eso. Entonces, eso es algo positivo" (hombre, Mitú-Vaupés, entrevista 6)

Hay también un asunto importante y es que el liderazgo da la posibilidad de generar transformaciones de las prácticas, porque hay convocatoria y poder de movilización. En síntesis, ese liderazgo se traduce en respeto "Fui a una reunión de docentes hace poco con el paro, no había uno que no hubiese sido mi estudiante, y el respeto que expresaban cuando yo tomaba la palabra, mi señora me decía: se callaron. Porque no se callan ante nadie" (hombre, Convención-Norte de Santander, 8,73). 
En síntesis, el reconocimiento está ligado a la categoría de capacidad y construcción de la identidad. El profesor se constituye no solo en su oficio sino en su ser, a través de la práctica misma que le permite declararse como capaz de hacer, de lograr, de transformar, de liderar (RICOEUR, 1996) y en ese despliegue se siente depositario de cumplir la misión educativa a la cual se siente llamado.

Y aparece entonces el correlato del testigo (RICOEUR, 1996), que reconoce los signos del cambio producido: los estudiantes de manera privilegiada, los padres de familia que se sienten agradecidos por los signos visibles de la formación en sus hijos, la comunidad local que aprecia en sus propias vidas los efectos transformadores del trabajo del profesor y expresan un agradecimiento expreso o tácito, los egresados que son motivo de orgullo por sus realizaciones personales y profesionales. Todo esto confirma el sentido del llamado a ser maestro, como fuente de identidad.

\subsection{La práctica pedagógica, motor de transformación}

Otra faceta muy importante del reconocimiento, que revelan los relatos de maestros y maestras es el poder de hacer con otros, y en esa acción encontrar que la práctica es posibilidad de transformación de las personas, en la práctica pedagógica es posible "tocar" el ser del otro y eso es posible verlo, percibirlo, lo que implica preparación y un cambio de los estilos autoritarios hacia unos más dialógicos. Esta acción transformadora pasa por lo más básico como enseñar a leer y escribir: "Lo más significativo que he tenido como maestra en todos estos años ha sido enseñarles a leer y escribir a muchos niños. Es lo máximo que hay. Cuando uno escucha al niño leer con soltura, la emoción es única. Y ese milagro lo veo todos los años" (mujer, Istmina-Chocó, entrevista 1). Pasa por ver los resultados que tienen los estudiantes que pueden ser motivados para aprender y llega a encontrarse con cambios significativos en la vida de las personas.

Hay un asunto particular que aparece en los relatos de los profesores y es el papel importante que tiene la práctica del maestro para construir esperanza en medio de las dificultades, para ayudar a que las ilusiones se conviertan en motores de trabajo, para enseñar a soñar, "Lo más significativo en mi vida como profesora ha sido lo de Carurú, enseñarles a soñar a los muchachos Cuando uno de verdad quiere lograr algo en los estudiantes con buena dedicación se puede, reflexividad narrativa sin ponerle tantas arandelas a las cosas" (mujer, Mitú-Vaupés, entrevista 5).

Por supuesto están también las posibilidades de transformación de la realidad a partir de su conocimiento, de cambio de situaciones que ayuden a mejorar las 
condiciones de vida de las personas, lo que sitúa al profesor más allá del aula y lo lleva a asumir un rol de liderazgo mediador. "El cambio solo es posible a través de la Educación y hay necesidad de movilizar el pensamiento, pienso que esa es la oportunidad de ser maestro" (mujer, Barbacoas-Nariño, entrevista 9).

Esa transformación también se refleja en los relatos de los profesores y las profesoras, en lo que podría denominarse los testimonios de los egresados. Algunos tienen que ver con los cargos de liderazgo que han alcanzado: alcaldes, concejales, secretarios de Educación. Otros tienen que ver el agradecimiento y la satisfacción que reciben al verlos profesionales, con sus proyectos de vida; se trata de la conformación de un vínculo que tiene algo de discipulado, de acompañamiento, "Uno dice: aquí está mi trabajo" (hombre, Istmina-Chocó, entrevista 2).

Por tratarse de profesores de escuelas normales es particularmente significativo el tema de la formación de maestros, de una parte, el tener la posibilidad de ver a muchos de ellos como compañeros en el municipio, o en la misma escuela, y además por la enorme responsabilidad social que eso implica, la formación de nuevas generaciones para cambiar la Educación. "Lo más significativo es saber que cada dos años gradúo una promoción de maestros. Le entrego a la sociedad un grupo de maestros que va a atender una nueva población de niños. Cada dos años ese es un momento de felicidad para mí" (mujer, Medellín-Antioquia, entrevista piloto).

Un sentimiento muy importante asociado al reconocimiento por la posibilidad de transformación es el del agradecimiento dado por estudiantes y padres de familia, una especie de testigo que da cuenta del alcance que tiene la práctica, un otro que otorga y constituye al maestro como transformador. Esa gratitud está relacionada con aprecio, cariño, confianza en el trabajo realizado, reconocimiento del esfuerzo realizado y en cierta medida eso confiere autoridad moral e influye en la propia imagen que el maestro construye de sí mismo "En la medida en que se avanza en este proceso los estudiantes empiezan a jugar un papel tan importante en la construcción de uno como maestro que por naturaleza uno dice me voy a preparar mejor" (hombre, Convención-Norte de Santander, entrevista 8).

En síntesis, la práctica se ubica en el mundo de la vida, ser, saber y hacer, que no se dan como universos escindidos sino como realidades que conviven en lo cotidiano en la tensión que Ricoeur (2004) plantea entre el horizonte de sentido y el hacer que logra pequeñas transformaciones. En síntesis, "supone pues poner en el centro la vida que se vive, lo que compone las vidas de cada uno, de cada una. Y supone poner en el centro de la experiencia educativa nuestras vidas 
compartidas con nuestros estudiantes, con aquellos de quienes asumimos una responsabilidad educativa" (CONTRERAS; PÉREZ, 2010, p. 35).

\subsection{El maestro, llamado a la misión educativa}

Un tercer aspecto respecto al reconocimiento es el hecho de que los relatos muestran que los profesores y las profesoras comprenden el oficio como el cumplimiento de una misión a la cual han sido llamados, lo que implica una actitud de entrega, una disposición especial, y eso constituye un don, un regalo, un privilegio y una distinción.

Una característica distintiva de esa misión, son los sujetos que le dan sentido, y el tipo de trabajo que se hace con ellos, que tiene que ver con iniciar a otros en un camino; ser una guía, dar orientación, indicar rumbos, mostrar otros mundos, proponer sentidos para la construcción de un proyecto de vida, lo que implica leer los contextos y comprenderlos, sin lo cual no es posible el aprendizaje (RICOEUR, 1996). Atañe también a enseñar a soñar, a tener esperanza, y por supuesto a ayudar, a ser solícito, a atender las necesidades de los estudiantes, "estar ahí con los que me necesitan, al lado de ellos. Ser maestro para ellos, ser parte de la solución a los problemas" (mujer, Convención-Norte de Santander, entrevista 7).

Otro rasgo es que esa misión implica un entregarse, un dar lo mejor que se tiene, desprenderse de sí mismo, "entregar el alma y el corazón" (hombre, Riosucio-Caldas, entrevista 14), tener un proceso de formación, esfuerzo y mejoramiento continuo, lo que conlleva compromiso con el trabajo y responsabilidad con las múltiples tareas que demanda la vocación, aunque no siempre encuentre agradecimiento por parte de la sociedad.

Finalmente, un rasgo muy particular de esa vocación es una especie de retribución que tienen los maestros y es la posibilidad de un aprendizaje permanente y creciente. Por ejemplo, trabajar con las comunidades permite insertarse en la cultura, conocer otras lenguas, vivir una permanente retroalimentación de saberes. El aprendizaje deviene también de sentirse retado a pensar los espacios de aprendizaje de una manera distinta, "Lo más importante de ser maestra es que he aprendido a rehacerme y pensarme la escuela todos los días, la escuela, la formación, y eso mantiene viva mi esperanza de maestra, me motiva, la llevo como una bandera pa' delante es que vamos" (mujer, Riosucio-Caldas, entrevista 13).

En síntesis, las capacidades para la práctica son un núcleo de valores, de habilidades innatas y aprendidas y un acervo de conocimientos que dan 
idoneidad, o un valor agregado para asumir la tarea máxima de hacerse profesor que se asume como una misión a la cual se es llamado. Se trata de una toma de conciencia de lo que se es y de lo que se ha logrado, de lo que es constitutivo y otorga el acceso a la práctica.

\subsection{Trabajar en la escuela normal da reconocimiento}

La escuela normal es reconocida en los territorios por su calidad académica; los padres de familia quieren que sus hijos estudien en esas instituciones, por lo tanto, ser profesor allí es tener un reconocimiento profesional, se trata de una transferencia de estatus de la organización a sus profesores que aparece de manera repetida en los relatos y que le permite sentirse un profesor especial :

Una cosa es trabajar en una institución educativa de formación básica y otra es trabajar en una escuela normal, eso lo tengo claro. Distintos compañeros que laboran en otras instituciones dicen que se nota desde el discurso oral y pedagógico que uno maneja en la práctica, esas son cosas que distinguen a un docente de una normal superior (hombre, Uribia-Guajira, entrevista 4).

El reconocimiento también está por el fuerte vínculo con la comunidad local y la capacidad de convocatoria y credibilidad que tienen las escuelas normales, lo que plantea un reto para el trabajo del profesor. De otra parte, está la atención especial que reciben los profesores en términos de formación continua, por hacer parte de las escuelas normales, "La Escuela Normal da muchos espacios que otro tipo de instituciones no tienen, hay mucho roce académico y pedagógico a nivel de país" (mujer, San Juan Nepomuceno, entrevista 11).

Por esa identificación tan grande con la escuela normal, las dificultades de la institución se sienten como propias; es común leer en los relatos y escuchar en las historias de los profesores la expresión "Me duele la escuela", que refleja un inmenso grado de personificación de la organización en una relación que configura la vida. Por eso entristece cuando hay oposición de un grupo o falta de apoyo.

También hay preocupación frente al prestigio de la escuela normal, por eso afanan a los maestros las divisiones internas en la institución que afectan el proceso formativo y la credibilidad externa de los egresados. Inquietan también a los maestros los problemas que tienen que ver con la planta física e incluso con la continuidad de las escuelas en el concierto nacional, 
Otra preocupación es la planta física, en este momento estamos hacinados en muchos espacios que no son muy acordes con las búsquedas de la Normal. Un temor es que se pierda un espacio que le ha contribuido tanto a la sociedad, que desaparezcan, habiendo contribuido tanto a la región (hombre, Riosucio-Caldas, entrevista 14).

Aunque de la misma forma hay que decir que esa identificación se da también en los logros de la escuela normal, en sus alegrías, que son también vividas por las comunidades locales,

Cuando la Escuela logra la acreditación previa en el 2003, eso hubo una fiesta, toda la comunidad estaba pendiente, el pueblo. Y cuando dan ese resultado allá, solo recuerdo que venía una banda tocando y todos los profesores adelante a festejar porque la Normal había sido acreditada (hombre, San Juan NepomucenoBolívar, entrevista 12).

En síntesis, la práctica pedagógica no se refiere a un sujeto individual sino a un sujeto que de algún modo ha sido constituido en el cruce de prácticas, saberes y una institución llamada escuela (ZULUAGA GARCÉS, 2005). El análisis de las prácticas permite caracterizar los modos del oficio de maestro según la hegemonía de los discursos circulantes.

\subsection{La identificación con los objetivos de la escuela da reconocimiento}

Un elemento que emerge con fuerza en la relación de los profesores con la escuela normal es la identificación con su objetivo de formadora de maestros y con el impacto social que producen, lo que los hace sentirse parte de una misión educativa que trasciende (GRUNDY, 1994). En muchos lugares se trata de auténticos oasis que además de Educación ofrecen muchos servicios de apoyo para las comunidades, un refugio, una fortaleza en tiempos de violencia

El restaurante escolar es un privilegio, porque se aprovecha ese momento para solventar esa necesidad que tienen los niños. Y con las niñas, es una fortaleza inmensa que ellas queden acá en el internado, a veces los papás las dejan aquí y se demoran para venir. Yo siento que aquí están cuidadas (mujer, Uribia-Guajira, entrevista 3). 
Una característica de la Escuela Normal es que siempre se ha sobrepuesto a las condiciones del contexto, nosotros logramos sobrevivir toda la época de violencia, a pesar de que nos desaparecieron dos rectores... (hombre, San Juan NepomucenoBolívar, entrevista 12).

En síntesis, la práctica se vuelve praxis. Orientada hacia el cambio social y la emancipación política y que implica una transformación de la conciencia, o sea, de los modos de ser y estar en el mundo.

\section{Conclusiones}

La categoría de reconocimiento encuentra una síntesis en el reconocimiento del liderazgo; ese despliegue de capacidad de los profesores y profesoras que es otorgado por las comunidades locales, los compañeros de trabajo y otros grupos de interacción social en los que actúan los maestros.

Asociado al liderazgo está la autoridad conferida que implica una responsabilidad mayor, la confianza en el poder del saber que le atribuyen las personas, la capacidad de tomar decisiones, el acontecimiento de ayudar a otros de "tocar el corazón"; emerge el campo de influencia que implica el oficio de maestro, el posicionamiento institucional y la posibilidad de transformar e innovar, que es uno de los aspectos más importantes de este reconocimiento que asume la forma de la solidaridad.

Aquí es importante decir que la práctica pedagógica como lugar de reconocimiento se vuelve mediación transformadora de los sujetos y de los contextos; la práctica es lugar para fabricar sueños, es un nacedero de esperanza, y eso está muy ligado a la conciencia de que ser profesor conlleva una misión que implica iniciar a otros en un camino que tiene horizonte y sentido y que aceptar ese trabajo implica entrega personal, una mística que hace parte de la identidad profesional.

Comprender esta conclusión abre un importante campo de investigación respecto al lugar que tiene la práctica como lugar de reconocimiento del maestro, la manera en la que se ve a sí mismo, se autorreferencia, se autodefine, y dialoga con la valoración social que el medio otorga al oficio.

Sería importante indagar sobre las historias de profesores en contextos urbanos, en las grandes ciudades, en colegios privados, en profesores universitarios, y en profesores que hasta ahora empiezan su trayectoria y están recién llegados 
a contextos institucionales, para establecer marcos comparativos respecto a las categorías que esta investigación ha identificado en profesores situados en contextos rurales impactados por el conflicto armado, para identificar recurrencias y diferencias que permitan construir un mapa que oriente los énfasis del reconocimiento y la identidad que deben estimular a las nuevas generaciones a optar por el trabajo docente. 


\section{Reconhecimento: uma característica das práticas pedagógicas em histórias de professores de escolas normais em contextos de conflito interno na Colômbia}

\section{Resumo}

Esse artigo tem como objetivo mostrar os sentidos das práticas pedagógicas narradas por professores de escolas normais localizadas em contextos educacionais impactados pelo conflito armado interno colombiano. A metodologia utilizada foi qualitativa com abordagem hermenêtica a partir da narrativa. O grupo de estudo consistia em 17 professores de 7 regiões diferentes da Colômbia afetadas pelo conflito armado interno colombiano. A prática pedagógica como lugar de reconhecimento, na perspectiva ricoeuriana, é uma dimensão central que é descrita nesse trabalho. É a categoria mais relevante que surge na maioria dos relatos dos professores e está ligada à capacidade e à configuração da identidade. O professor se forma pela prática, o que lhe permite se declarar capaz de fazer, de realizar, de transformar, de liderar, e que, nesse desdobramento se sinta depositário da missão de educar.

Palavras-chave: Reconhecimento. Prática Pedagógica. Pesquisa Narrativa. Pedagogia Narrativa.

\section{Acknowledgement: a characteristic of pedagogical practices in stories of teachers from teacher training colleges in contexts of internal armed conflict in Colombia}

\section{Summary}

This essay aims to show the meanings of the pedagogical practices narrated by teachers of teacher training colleges located in educational contexts impacted by the Colombian internal armed conflict. The methodology used was of a qualitative research with a hermeneutical approach from narrative. The study group was composed of 17 teachers from seven different regions in Colombia affected by the Colombian armed internal conflict. Educational practices as a place of acknowledgment, in the Ricoeurian perspective, are a central dimension described in this work. It is the most relevant category that emerges in all the stories of the teachers and that is linked to the capacity and setting of identity. The teacher forms himself through practice, which allows him to declare himself capable of doing, of achieving, of transforming, of leading, and that in this deployment he feels he is the depositary of the educational mission.

Keywords: Acknowledgment. Educational Practices. Narrative Research. Narrative Pedagogy. 


\section{REFERENCIAS}

ATKINSON, P. The life story interview as a bridge in narrative inquiry. In: CLANDININ, D. J. (ed.). Handbook of narrative inquiry: mapping a methodology. Thousand Oaks: Sage, 2007. p. 224-245.

BEAUCHAMP, C.; THOMAS, L. Understanding teacher identity: an overview of issues in the literature and implications for teacher education. Cambridge Journal of Education, Cambridge, v. 39, n. 2, p. 175-189, 2009. http://dx.doi.org/10.1080/03057640902902252.

BOLÍVAR, A. Ciclo de vida profesional de profesores y profesoras de secundaria: desarrollo e itinerarios de formación. In: CERDÁN, J.; GRAÑERAS, M. (eds.). Investigación sobre el profesorado (II) 1993-1997. Madrid: Ministerio de Educación y Ciencia; 1997. p. 163-191.

CLANDININ, J.; ROSIEK, J. Mapping a landscape of narrative inquiry. In: CLANDININ, D. J. (ed.). Handboock of narrative inquiry: mapping a methodology. Thousand Oaks: Sage, 2007. p. 35-75.

CONTRERAS, J.; PÉREZ, N. Investigar la experiencia educativa. Madrid: Morata, 2010.

FERGUSON, M. R. Finding a home abroad with "Eveline": using narrative inquiry to establish a sense of place for a western teacher in a foreign and multicultural context. Journal of Studies in International Education, [s. 1.], v. 15, n. 1, p. 25-40, June 2011. https://doi.org/10.1177/1028315309337928

GAJARDO-ASBUN, K. Estado del arte sobre identidad docente: investigación de experiencias de profesores en formación y en ejercicio. $I E$ Revista de Investigación Educativa de la REDIECH, Chihuahua, v. 10, n. 18, p. 79 - 93, abr. 2019. https://doi.org/10.33010/ie_rie_rediech.v10i18.217

GRUNDY, S. Teoría y praxis del currículo. Madrid: Morata. 1994.

HOWARD, K. Temporal landscapes of morality in narrative: student evaluation in a Thai parent-teacher conference. Discourse \& Society, London, v. 19, n. 2, p. 163-186, Mar. 2008. https://doi.org/10.1177/0957926507085951

KEMMIS, S. From popular science to public spheres. In: SOUZA, J. F.; O'HANLON, C.; FALS-BORDA, O. Investigación acción y educación en contextos de pobreza: un homenaje a la vida y obra del maestro Orlando Fals Borda. Bogotá: Unisalle, 2007. p. 101-117. 
MOLANO, M. Narrativas de las prácticas pedagógicas en contextos impactados por el conflicto interno armado colombiano: relatos de maestros de escuelas normales superiores. Tesis (Doctorado en Educación y Sociedad) - Universidad de la Salle, Bogotá, Colombia, 2019. Disponible en: Retrieved from https://ciencia.lasalle.edu.co/doct_educacion_sociedad/31. Acceso en: 17 ene. 2020.

MOLANO, M.; BAQUERO, P. Dirigir y tutorar proyectos de investigación narrativa: experiencias, reflexiones y preguntas. Actualidades Pedagógicas, Bogotá, n. 54, jul.-dic. 2009.

MORTOLA, G. Una aproximación narrativa a la construcción de la identidad laboral docente. Educación, Lenguaje y Sociedad, La Pampa, v. 4, n. 4, p. 83-104, dic. 2006.

NESPOR, J.; BARYLSKE, J. Narrative discourse and teacher knowledge. American Educational Research Journal, Washington, v. 28, n. 4, p. 805-823, dec. 1991. https://doi.org/10.3102/00028312028004805

OLSHTAIN, E.; KUPFERBERG, I. Reflective-narrative discourse of FL teachers exhibits profesional knowledge. Language Teaching Research, [s. 1.], v. 2, n. 3, p. 185-202, jul. 1998. https://doi.org/10.1177/136216889800200302

QUINTERO, M. Usos de las narrativas, epistemologías y metodologías: aportes para la investigación. Bogotá: Editorial Universidad Distrital Francisco José de Caldas, 2018.

RESTREPO, B. Un modelo de capacitación de maestros en servicio, basado en la investigación-acción pedagógica. In: ÁVILA, R. La investigación-acción pedagógica: experiencias y lecciones. Bogotá: Antrophos, 2006. p. 39-68.

RICOEUR, P. Sí mismo como otro. México: Siglo XXI, 1996.

RICOEUR, P. Volverse capaz, ser reconocido [Discurso recepción del premio Kluge]. Washington: Biblioteca del Congreso de los Estados Unidos, 2004.

ROSIEK, J.; ATKINSON, B. The inevitability and importance of genres in narrative research on teaching practice. Qualitative Inquiry, [s. 1.], v. 13, n. 4, p. 499-521, May 2007. https://doi.org/10.1177/1077800406297669

TULVISTE, P. Tailoring identities. Culture \& Psychology, London, v. 17, n. 2, p. 217-221, June 2011. https://doi.org/10.1177/1354067X11398316 
VALENCIA, L.; AVILA, A. Los retos del postconflicto: justicia, seguridad y mercados ilegales. Bogotá: Grupo Zeta, 2016.

VANDEYAR, T. A window to teachers' ICT practices: Discerning between teaching and the complex science of pedagogy. Ensaio: Avaliação e Politicas Públicas em Educação, Rio de Janeiro, v. 28, n. 109, Oct.-Dec. 2020. https://doi.org/10.1590/s0104-40362020002802388

VENÄLÄINEN, R. What do we know about instructional time use in Mali? World Bank, 2009. Disponible in: http://www-wds.worldbank.org/external/ default/WDSContentServer/WDSP/IB/2009/02/11/000333037_2009021100 4247/Rendered/PDF/473280WP0Box33101OFFICIAL0USE0ONLY1.pdf. Acceso en: 11 oct. 2011.

ZULUAGA GARCÉS, O. L. Foucault: una lectura desde la práctica pedagógica. In: ZULUAGA GARCÉS, O. L.; ZULLAGA DE ECHEVERRY, O. L. Foucault, la pedagogía y la educación: pensar de otro modo. Bogotá: Universidad Pedagógica Nacional, 2005. p. 11-37.

ZULUAGA NIETO, J. Situación actual y perspectivas de la guerra interna. Cahiers des Amériques Latines, Paris, n. 71, 2012. https://doi.org/10.4000/cal.2704

\section{Información de los autores}

Milton Molano Camargo: Doctor en Educación y Sociedad de la Universidad de La Salle, Bogotá, Colombia. Docente de la misma Universidad. Contacto: mmolano@lasalle.edu.co

iD https://orcid.org/0000-0003-1330-7709

Felipe Andrés Aliaga Sáez: Doctor en sociología y procesos políticos contemporáneos por la Universidad de Santiago de Compostela. Docente investigador de la Facultad de Sociología de la Universidad Santo Tomás, Colombia, y Tutor del Doctorado en Educación y Sociedad de la Universidad de La Salle, Bogotá, Colombia. Contacto: felipealiaga@ usantotomas.edu.co

iD https://orcid.org/0000-0003-4635-1132 